\title{
Interaction Between Sex and Left Ventricular Reverse Remodeling and Its Association with Outcomes After Transcatheter Aortic Valve Implantation
}

Jurrien H. Kuneman ( $\boldsymbol{\sim}$ j.h.kuneman@lumc.nl )

Leiden University Medical Center: Leids Universitair Medisch Centrum https://orcid.org/0000-00032162-2768

\section{Steele C. Butcher}

Leiden University Medical Center: Leids Universitair Medisch Centrum Jan Stassen

Leiden University Medical Center: Leids Universitair Medisch Centrum

\section{Gurpreet K. Singh}

Leiden University Medical Center: Leids Universitair Medisch Centrum

Stephan M. Pio

Leiden University Medical Center: Leids Universitair Medisch Centrum

\section{Frank van der Kley}

Leiden University Medical Center: Leids Universitair Medisch Centrum

\section{Nina Ajmone Marsan}

Leiden University Medical Center: Leids Universitair Medisch Centrum

Juhani Knuuti

Turku PET Centre: Turun PET keskus

Jeroen J. Bax

Leiden University Medical Center: Leids Universitair Medisch Centrum

\section{Victoria Delgado}

Leiden University Medical Center: Leids Universitair Medisch Centrum https://orcid.org/0000-00029841-2737

\section{Research Article}

Keywords: aortic stenosis, left ventricular reverse remodeling, sex differences, transcatheter aortic valve implantation, echocardiography

Posted Date: January 31st, 2022 
DOI: https://doi.org/10.21203/rs.3.rs-1278635/v1

License: (c) (1) This work is licensed under a Creative Commons Attribution 4.0 International License. Read Full License 


\section{Abstract \\ Purpose}

Women with severe aortic stenosis (AS) have better long-term prognosis after transcatheter aortic valve implantation (TAVI) compared to men. Whether this is caused by sex-related differences in left ventricular (LV) reverse remodeling after TAVI is unknown.

\section{Methods}

Patients with severe AS who underwent transfemoral TAVI between 2007 and 2018 were selected. LV dimensions, volumes, and ejection fraction (LVEF) were assessed by transthoracic echocardiography before TAVI and at 6 and 12 months follow-up after TAVI. LV reverse remodeling was defined as the percentual LV mass index (LVMi) reduction compared to baseline. The primary outcome was all-cause mortality.

\section{Results}

A total of 459 patients ( $80 \pm 8$ years; $52 \%$ male) were included. At 6 and 12 months follow-up, both sexes showed significant reductions in LV volumes and LVMi accompanied by improvement in LVEF, without significant differences between the sexes over time. During a median follow-up of 2.8 [IQR 1.9-4.3] years, $181(39 \%)$ patients died. Women showed better outcomes compared to men (log-rank $p=0.024)$. In addition, male sex was independently associated with all-cause mortality in multivariable Cox regression (HR 1.423, 95\% Cl: 1.039-1.951, p=0.028). No association was observed between the interaction of percentual LVMi reduction and sex with outcomes $(p=0.64)$.

\section{Conclusion}

Men and women with severe AS had similar improvement in LVEF, and similar reduction in LV volumes and LVMi at 6 and 12 months after TAVI. Women showed better survival after TAVI as compared to men. The superior outcomes noted in women after TAVI are not associated with sex differences in LV reverse remodeling.

\section{Introduction}

Untreated severe aortic stenosis (AS) has a poor prognosis [1, 2]. Aortic valve replacement is an effective therapy and significantly reduces morbidity and mortality in patients with severe AS $[3,4]$. Transcatheter aortic valve implantation (TAVI) has emerged as a valid alternative to surgical aortic valve replacement for patients with AS who are inoperable or have high operative risk [5]. Moreover, recent studies showed promising results of TAVI in patients at intermediate and low surgical risk [6-8]. Interestingly, the survival 
benefit of TAVI differs between men and women [9-11]. Women with severe AS have shown an increased risk of complications during TAVI, but have better survival after TAVI compared to men $[12,13]$.

Differences in left ventricular (LV) reverse remodeling between men and women may be a potential explanation for the sex-related outcome differences after TAVI. The LV remodeling pattern in response to the pressure overload caused by severe AS may differ between men and women [14, 15]. However, little is known on how the regression of LV mass and changes in LV geometry occur after TAVI.

Some studies suggested the presence of sex-related differences in LV reverse remodeling after TAVI, including more LV mass regression and greater improvement in LV ejection fraction (LVEF) in women versus men [16-19]. However, the association between the different LV reverse remodeling patterns after TAVI between men and women as well as the differences in outcomes have not been fully elucidated. Accordingly, the aims of this study were to examine 1) sex differences in LV reverse remodeling after TAVI and 2) to evaluate whether these differences are associated with the different outcomes after TAVI.

\section{Methods}

\section{Study population}

Patients with severe AS treated with TAVI at the Leiden University Medical Center (Leiden, The Netherlands) between 2007 and 2018 were evaluated in this retrospective analysis. Exclusion criteria were: bicuspid aortic valve anatomy, transapical TAVI or a valve-in-valve procedure, or the lack of echocardiographic data at baseline and/or follow-up. All patients underwent standard routine transthoracic echocardiography. Demographic and clinical characteristics at the time of TAVI were obtained from the electronic patient records (EPD-vision, Leiden University Medical Center, Leiden, The Netherlands and HiX version 6.1, ChipSoft B.V., Amsterdam, The Netherlands). Clinical characteristics included cardiac risk factors, comorbidities, previous coronary revascularization, and medication use. The estimated glomerular filtration rate (eGFR) was calculated using the Chronic Kidney Disease Epidemiology Collaboration (CKD-EPI) formula, and renal impairment was defined as eGFR $<60$ $\mathrm{mL} / \mathrm{min} / 1.73 \mathrm{~m}^{2}$. This retrospective analysis complies with the Declaration of Helsinki and was approved by the institutional review board which waived the need for written informed consent due to the retrospective study design.

\section{Transcatheter aortic valve procedure}

TAVI eligibility and feasibility as well as decision-making on the access route and valve type were decided by the local heart team. Transcatheter heart valve size was selected based on multidetector-row computed tomography measurements of the aortic annulus, as previously described [20]. The TAVI procedure was performed according to standard practice [21]. Balloon- and self-expandable valves that were used include Edwards SAPIEN, SAPIEN XT, SAPIEN 3 (Edwards Lifesciences, Irvine, CA, USA) and Medtronic CoreValve Evolut R and Evolut Pro (Medtronic, MN, Minnesota, USA).

\section{Echocardiography}


Transthoracic 2-dimensional echocardiographic examinations were performed with patients at rest and the data were acquired from the parasternal, apical, and subcostal views. All echocardiographic examinations were acquired by experienced echocardiographers using commercially available ultrasound systems (Vivid-7, E9 or E95, General Electric Vingmed, Horten, Norway) equipped with 3.5 MHz or M5S transducers. All images including two-dimensional, M-mode, and Doppler data were digitally stored for offline analysis using commercially available software (EchoPac version 113.0.3 and 203; GE Medical Systems, Horten, Norway). The echocardiographic examinations were performed before TAVI (aortic valve hemodynamics, LV function, LV dimensions) and during routine clinical follow-up: immediately after TAVI (prosthetic valve gradients) and at 6 and 12 months after TAVI (prosthetic valve gradients, LV function, LV dimensions), and were reported according to current recommendations [22, 23]. LV dimensions (enddiastolic diameter, intraventricular septum thickness, posterior wall thickness) were obtained in parasternal long-axis views at end-diastole. LV mass was calculated using the Devereux formula and was indexed to body surface area [23]. In addition, the LV remodeling patterns were determined as defined by the relative wall thickness and the presence of LV hypertrophy (defined by a LV mass index $>95 \mathrm{~g} / \mathrm{m}^{2}$ in women and $>115 \mathrm{~g} / \mathrm{m}^{2}$ in men): normal geometry (absence of LV hypertrophy and relative wall thickness $\leq 0.42$ ), concentric remodeling (absence of LV hypertrophy and relative wall thickness $>0.42$ ), concentric hypertrophy (LV hypertrophy and relative wall thickness $>0.42$ ), and eccentric hypertrophy (LV hypertrophy and relative wall thickness $\leq 0.42)[23,24]$. AS severity was determined before TAVI. Aortic valve area was calculated using the continuity equation and indexed to body surface area (AVAi). Severe aortic valve stenosis was defined as an aortic valve area $<1.0 \mathrm{~cm}^{2}$ or AVAi $<0.6 \mathrm{~cm}^{2} / \mathrm{m}^{2}$, mean transvalvular pressure gradient $\geq 40 \mathrm{mmHg}$, and a peak aortic jet velocity $\geq 4 \mathrm{~m} / \mathrm{s}$ [25]. Peak and mean transvalvular gradients were calculated from continuous wave Doppler recordings of the apical 3- or 5-chamber views according to Bernoulli equation [22]. LV volumes (end-diastolic and end-systolic) were measured in the apical 2-and 4-chamber views and indexed to body surface area [23]. Body surface area was considered consistent between each follow-up visit. LVEF was estimated using Simpsons' biplane method [23]. The presence of post-procedural aortic regurgitation and paravalvular leakage were detected and severity was graded according to current recommendations: mild (grade 1), moderate (grade 2), moderate to severe (grade 3), and severe (grade 4) [26]. Significant paravalvular leakage was defined by a grade $\geq 2$.

\section{Clinical endpoints and follow-up}

Changes in prosthetic valve gradients, LV function, and LV mass index over time are presented in absolute numbers and expressed as percentual reduction compared to baseline (pre-TAVI). Additional echocardiographic endpoints to identify sex-related differences in the magnitude of LV reverse remodeling and abnormal prosthetic valve gradients during 1-year echocardiographic follow-up included: a) $\geq 10 \%$ reduction in LV end-diastolic volume index (LVEDVi), b) $\geq 10 \%$ reduction in LV end-systolic volume index (LVESVi), c) $\geq 10 \%$ LV mass index reduction, d) $\geq 20 \%$ LV mass index reduction, e) mean gradient $\geq 20 \mathrm{mmHg}$, and $\mathrm{f}$ ) reduction in LVEF $\geq 5 \%$.

Patients were followed up for the occurrence of the primary endpoint of all-cause mortality. Survival time was recorded from the final follow-up echocardiographic examination at 6 or 12 months after TAVI and 
was restricted to 6 years. Data on mortality were collected from the departmental electronic patient files which were linked with the Social Security Death Index. Follow-up data were acquired for all patients.

\section{Statistical analysis}

Continuous variables following a normal distribution are presented as mean \pm standard deviation and were compared using the independent Student t-test. Non-normally distributed continuous variables are presented as median with $25 \%-75 \%$ interquartile range and were compared using the Mann-Whitney U test. Distribution of continuous variables was evaluated using histograms and Q-Q plots. Categorical variables are presented as absolute numbers and percentages and were compared using the $\chi 2$ test or Fisher's exact test. General linear models with repeated measures analysis were used to evaluate changes in echocardiographic variables over time and to test differences between men and women over time. The Greenhouse-Geisser correction was used if the sphericity assumption was violated. Additional analyses were performed to correct for the potential confounding effect of age, body mass index, hypertension, diabetes mellitus, coronary artery disease, previous myocardial infarction, concomitant moderate or severe mitral regurgitation or aortic regurgitation, moderate or severe paravalvular leakage, pre-TAVI LVEF, and AVAi on the change of the LV parameters over time, and were included as covariates in the general linear models [27]. Kaplan-Meier curves were generated to estimate the cumulative survival rates of allcause mortality and the log-rank test was used to compare the survival between men and women. Multivariable Cox proportional hazards regression analysis was used to evaluate the association of sex and percentual LV mass index reduction with all-cause mortality. Potential confounders including age, cardiac risk factors, coronary artery disease, previous myocardial infarction, previous stroke/transient ischemic attack, peripheral vascular disease, chronic obstructive pulmonary disease, chronic kidney disease, and pre-TAVI LVEF were incorporated in the multivariable Cox proportional hazard model. Additional multivariable Cox proportional hazard regression models were used to test the association between percentual LV mass index reduction and sex (as interaction term) on outcomes. Hazard ratios (HR) and 95\% confidence intervals (Cl) were calculated and reported. A two-sided $p$-value $<0.05$ was considered significant. Data analysis was performed with SPSS version 25.0 (IBM SPSS Statistics, IBM Corporation, Armonk, New York, USA).

\section{Results}

\section{Patient and procedural characteristics}

A total of 459 patients (age $80 \pm 7$ years, $52 \%$ males) with severe AS who underwent transfemoral TAVI and had echocardiographic follow-up were included in the analysis. Baseline (pre-TAVI) demographic and clinical characteristics of the overall population and according to sex are presented in Table 1. Women were slightly but significantly older compared to men at the time of TAVI. Men had more frequent history of hypercholesterolemia and concomitant coronary artery disease as well as previous myocardial infarction and coronary revascularization. Accordingly, men were more frequently using statins and antiplatelet therapy. 
Table 1

Baseline demographical and clinical characteristics of the overall population and according to sex.

\begin{tabular}{|c|c|c|c|c|}
\hline Variable & $\begin{array}{l}\text { Overall } \\
\text { population } \\
\mathrm{n}=459\end{array}$ & $\begin{array}{l}\text { Men } \\
n=240\end{array}$ & $\begin{array}{l}\text { Women } \\
\mathrm{n}=219\end{array}$ & $\begin{array}{l}\text { p- } \\
\text { value }\end{array}$ \\
\hline Age, years & $80 \pm 7$ & $80 \pm 7$ & $81 \pm 7$ & 0.006 \\
\hline Body Surface Area, $\mathrm{m}^{2}$ & $1.87 \pm 0.21$ & $1.98 \pm 0.17$ & $1.74 \pm 0.16$ & $<0.001$ \\
\hline Body mass index, $\mathrm{kg} / \mathrm{m}^{2}$ & $26.7 \pm 4.6$ & $26.8 \pm 3.9$ & $26.6 \pm 5.3$ & 0.66 \\
\hline EUROSCORE & $12.6[8.6-20.2]$ & $\begin{array}{l}15.5[9.0- \\
24.8]^{-}\end{array}$ & $\begin{array}{l}14.5[10.1- \\
22.3]\end{array}$ & 0.85 \\
\hline eGFR (CKD-EPI), ml/min/1.73 m² & $60 \pm 20$ & $60 \pm 21$ & $61 \pm 19$ & 0.66 \\
\hline Hypertension, n (\%) & $322(73)$ & $175(73)$ & $157(72)$ & 0.83 \\
\hline Hypercholesterolemia, n (\%) & $278(61)$ & $156(65)$ & $122(56)$ & 0.048 \\
\hline Diabetes mellitus, n (\%) & $138(30)$ & $80(33)$ & $58(27)$ & 0.11 \\
\hline History of smoke habit, n (\%) & $67(15)$ & $41(17)$ & $26(12)$ & 0.12 \\
\hline CAD, n (\%) & $296(65)$ & $178(74)$ & $118(54)$ & $<0.001$ \\
\hline Previous myocardial infarction, n (\%) & $87(19)$ & $58(24)$ & $29(13)$ & 0.003 \\
\hline Previous revascularization, n (\%) & $243(53)$ & $156(65)$ & $87(40)$ & $<0.001$ \\
\hline $\mathrm{PCl}$ & $143(31)$ & $80(33)$ & $63(29)$ & $<0.001$ \\
\hline CABG & $100(22)$ & $76(32)$ & $24(11)$ & \\
\hline \multicolumn{5}{|l|}{ NYHA classification, n (\%) } \\
\hline $\mid-I I$ & $168(37)$ & $94(39)$ & $74(34)$ & \multirow[t]{2}{*}{0.23} \\
\hline III-IV & $291(63)$ & $146(61)$ & $145(66)$ & \\
\hline Previous stroke/TIA, n (\%) & $87(19)$ & $44(19)$ & $43(20)$ & 0.74 \\
\hline Peripheral vascular disease, $\mathrm{n}(\%)$ & $92(20)$ & $54(23)$ & $38(17)$ & 0.17 \\
\hline Atrial fibrillation, n (\%) & $114(25)$ & $68(28)$ & $46(21)$ & 0.070 \\
\hline $\begin{array}{l}\text { Chronic obstructive pulmonary disease, } \\
\mathrm{n}(\%)\end{array}$ & $88(19)$ & $50(21)$ & $38(17)$ & 0.35 \\
\hline
\end{tabular}

Data are presented as mean \pm SD, median [25-75\% interquartile range] and $n(\%)$. ACE-I = angiotensinconverting enzyme, ARB II = angiotensin-II receptor blocker, CABG = coronary artery bypass grafting, $\mathrm{CAD}=$ coronary artery disease, $\mathrm{CKD}-\mathrm{EPI}=$ chronic kidney disease epidemiology collaboration, eGFR = estimated glomerular filtration rate, MR = mineralocorticoid receptor, NYHA = New York Heart Association, $\mathrm{PCl}=$ percutaneous coronary intervention, $\mathrm{TIA}=$ transient ischemic attack. 


\begin{tabular}{|c|c|c|c|c|}
\hline Variable & $\begin{array}{l}\text { Overall } \\
\text { population } \\
n=459\end{array}$ & $\begin{array}{l}\text { Men } \\
n=240\end{array}$ & $\begin{array}{l}\text { Women } \\
\mathrm{n}=\mathbf{2 1 9}\end{array}$ & $\begin{array}{l}\mathrm{p}- \\
\text { value }\end{array}$ \\
\hline \multicolumn{5}{|l|}{ Medication, n (\%) } \\
\hline Beta-blocker & $272(60)$ & $142(59)$ & $130(60)$ & 0.87 \\
\hline ACE-I/ARB II & $239(52)$ & $120(50)$ & $119(55)$ & 0.30 \\
\hline Calcium antagonist & $107(23)$ & $60(25)$ & $47(22)$ & 0.40 \\
\hline Diuretics & $255(56)$ & $129(54)$ & $126(58)$ & 0.35 \\
\hline MR antagonist & $50(11)$ & $35(15)$ & $15(7)$ & 0.009 \\
\hline Statins & $285(62)$ & $167(70)$ & $118(54)$ & 0.001 \\
\hline Antiplatelet & $275(60)$ & $159(66)$ & $116(53)$ & 0.004 \\
\hline Anticoagulation & $169(38)$ & $97(41)$ & $72(34)$ & 0.13 \\
\hline \multicolumn{5}{|c|}{$\begin{array}{l}\text { Data are presented as mean } \pm \text { SD, median }[25-75 \% \text { interquartile range] and } n(\%) \text {. ACE-I = angiotensin- }- \\
\text { converting enzyme, ARB } I I=\text { angiotensin-II receptor blocker, } \mathrm{CABG}=\text { coronary artery bypass grafting } \\
\mathrm{CAD}=\text { coronary artery disease, CKD-EPI = chronic kidney disease epidemiology collaboration, eGFR = } \\
\text { estimated glomerular filtration rate, } \mathrm{MR}=\text { mineralocorticoid receptor, NYHA = New York Heart } \\
\text { Association, } \mathrm{PCI}=\text { percutaneous coronary intervention, } \mathrm{TIA}=\text { transient ischemic attack. }\end{array}$} \\
\hline
\end{tabular}

Pre-TAVI echocardiographic data are presented in Table 2. There were no significant differences between men and women regarding the echocardiographic parameters that define severe AS. LV volumes were significantly larger in men versus women. In addition, LV mass index was significantly larger in men versus women before TAVI $\left(125 \pm 32 \mathrm{~g} / \mathrm{m}^{2}\right.$ vs. $112 \pm 33 \mathrm{~g} / \mathrm{m}^{2}$, respectively, $\left.\mathrm{p}<0.001\right)$. Concentric hypertrophy was the most frequently observed LV remodeling pattern before TAVI (47\%), followed by concentric remodeling (29\%), eccentric hypertrophy (16\%), and normal geometry (8\%), and the distribution of the LV remodeling patterns was comparable between the sexes. 
Table 2

Echocardiographic data before transcatheter aortic valve implantation.

\begin{tabular}{|c|c|c|c|c|}
\hline Variable & $\begin{array}{l}\text { Overall population } \\
\mathrm{n}=459\end{array}$ & $\begin{array}{l}\text { Men } \\
n=240\end{array}$ & $\begin{array}{l}\text { Women } \\
\mathrm{n}=219\end{array}$ & p-value \\
\hline AV peak gradient, $\mathrm{mmHg}$ & $65 \pm 24$ & $63 \pm 22$ & $68 \pm 26$ & 0.047 \\
\hline $\mathrm{AV}$ mean gradient, $\mathrm{mmHg}$ & $41 \pm 16$ & $41 \pm 15$ & $42 \pm 17$ & 0.36 \\
\hline AVAi, $\mathrm{cm}^{2} / \mathrm{m}^{2}$ & $0.41 \pm 0.12$ & $0.41 \pm 0.14$ & $0.41 \pm 0.13$ & 0.72 \\
\hline LVEDVi, ml/m² & 43 [36-55] & 48 [39-59] & 39 [33-50] & $<0.001$ \\
\hline LVESVi, ml/m² & $20[16-29]$ & $22[17-31]$ & 17 [14-25] & $<0.001$ \\
\hline $\mathrm{SVi}, \mathrm{ml} / \mathrm{m}^{2}$ & $39 \pm 13$ & $39 \pm 13$ & $38 \pm 12$ & 0.40 \\
\hline LVEF, \% & $51 \pm 10$ & $50 \pm 10$ & $52 \pm 11$ & 0.065 \\
\hline LV mass index, $\mathrm{g} / \mathrm{m}^{2}$ & $119 \pm 33$ & $125 \pm 32$ & $112 \pm 33$ & $<0.001$ \\
\hline LVEDD, mm & $47.6 \pm 7.3$ & $49.9 \pm 7.0$ & $45.2 \pm 7.0$ & $<0.001$ \\
\hline IVST, mm & $13.2 \pm 1.8$ & $13.5 \pm 1.8$ & $13.0 \pm 1.8$ & 0.001 \\
\hline PWT, mm & $12.1 \pm 2.2$ & $12.5 \pm 2.1$ & $11.7 \pm 2.1$ & $<0.001$ \\
\hline RWT & $0.51 \pm 0.13$ & $0.50 \pm 0.12$ & $0.52 \pm 0.14$ & 0.13 \\
\hline \multicolumn{5}{|l|}{ LV remodeling pattern } \\
\hline Normal geometry & $37(8)$ & $24(10)$ & $13(6)$ & \multirow[t]{4}{*}{0.19} \\
\hline Concentric remodeling & $133(29)$ & $70(29)$ & $63(29)$ & \\
\hline Concentric hypertrophy & $215(47)$ & $113(48)$ & $102(46)$ & \\
\hline Eccentric hypertrophy & $72(16)$ & $31(13)$ & $41(19)$ & \\
\hline \multicolumn{5}{|c|}{$\begin{array}{l}\text { Data are presented as mean } \pm \text { SD, median }[25-75 \% \text { interquartile range] and } \mathrm{n}(\%) \text {. AV = aortic valve, } \\
\text { AVAi = indexed aortic valve area, IVST = intraventricular septum thickness, LV = left ventricular, } \\
\text { LVEDVi = left ventricular end-diastolic volume index, LVEDD = left ventricular end-diastolic diameter, } \\
\text { LVEF = left ventricular ejection fraction, LVESVi = left ventricular end-systolic volume index, PWT = } \\
\text { posterior wall thickness, RWT = relative wall thickness, } S V i=\text { stroke volume index. }\end{array}$} \\
\hline
\end{tabular}

All patients underwent TAVI via the transfemoral approach. The majority of patients received balloonexpandable valves: Edwards SAPIEN 3 (57\%), SAPIEN XT (10\%), and SAPIEN (8\%). Self-expandable valves were used in $19 \%$ of patients. Prosthesis size ranged from $20 \mathrm{~mm}$ to $31 \mathrm{~mm}$, with $26 \mathrm{~mm}$ being most frequently used in 183 patients (40\%). 
Changes in LV volumes and LV mass index as well as LVEF over time, are displayed in Figure 1. At 6 and 12 months after TAVI, both men and women showed a significant reduction in LVEDVi (from 48 [39-59] $\mathrm{ml} / \mathrm{m}^{2}$ to 44 [37-55] $\mathrm{ml} / \mathrm{m}^{2}$ at 6 months and to 43 [34-53] $\mathrm{ml} / \mathrm{m}^{2}$ at 12 months after TAVl; and from 39 [33-50] $\mathrm{ml} / \mathrm{m}^{2}$ to 38 [31-46] ml/m $\mathrm{m}^{2}$ at 6 months and to 36 [28-43] $\mathrm{ml} / \mathrm{m}^{2}$ at 12 months, respectively, both $\mathrm{p}<0.001$ ) and in LVESVi (men: from 22 [17-31] $\mathrm{ml} / \mathrm{m}^{2}$ to 20 [16-27] $\mathrm{ml} / \mathrm{m}^{2}$ at 6 months and to 19 [1526] $\mathrm{ml} / \mathrm{m}^{2}$ at 12 months after TAVl; women: from 17 [14-25] $\mathrm{ml} / \mathrm{m}^{2}$ to 16 [13-21] $\mathrm{ml} / \mathrm{m}^{2}$ at 6 months and to $15[11-20] \mathrm{ml} / \mathrm{m}^{2}$ at 12 months, respectively, both $\left.\mathrm{p}<0.001\right)$, without significant differences between the sexes over time ( $p$ for interaction $=0.51$ and 0.43 , respectively). Moreover, LV mass index regressed significantly in both groups during follow-up (men: from $125 \pm 32 \mathrm{~g} / \mathrm{m}^{2}$ to $110 \pm 27 \mathrm{~g} / \mathrm{m}^{2}$ at 6 months and to $104 \pm 25 \mathrm{~g} / \mathrm{m}^{2}$ at 12 months after TAVI, $p<0.001$; women: from $112 \pm 33 \mathrm{~g} / \mathrm{m}^{2}$ to $99 \pm 24$ $\mathrm{g} / \mathrm{m}^{2}$ at 6 months and to $93 \pm 26 \mathrm{~g} / \mathrm{m}^{2}$ at 12 months, $\mathrm{p}<0.001 ; \mathrm{p}$ for interaction=0.44). Additionally, LVEF improved significantly in both groups during follow-up, without significant differences between men and women over time (from $50 \pm 10 \%$ to $53 \pm 9 \%$ at 6 months and to $53 \pm 10 \%$ at 12 months after TAVI in men, $p<0.001$ and from $52 \pm 11 \%$ to $55 \pm 9 \%$ at 6 months and to $56 \pm 9 \%$ at 12 months in women, $p<0.001 ; p$ for interaction=0.30). Similar results were observed when adjusting for potential confounders of LV reverse remodeling: no significant differences between men and women over time were observed in terms of improvement in LVEF and reduction in indexed LV volumes and LV mass. The distribution of the LV remodeling patterns for men and women before TAVI and at 6 and 12 months follow-up are displayed in Figure 3.

Changes in aortic valve prosthetic valve gradients over time are displayed in Figure 2. Aortic valve peak gradient decreased significantly after TAVI, without significant differences between men and women over time (men: from $63 \pm 22 \mathrm{mmHg}$ to $17 \pm 6 \mathrm{mmHg}$ immediately after TAVI and to $17 \pm 7 \mathrm{mmHg}$ at 6 months and to $17 \pm 8 \mathrm{mmHg}$ at 12 months after TAVI, $p<0.001$; women: from $68 \pm 26 \mathrm{mmHg}$ to $18 \pm 8 \mathrm{mmHg}$ immediately after TAVI and to $17 \pm 8 \mathrm{mmHg}$ at 6 months and to $18 \pm 8 \mathrm{mmHg}$ at 12 months, $p<0.001$, respectively; $p$ for interaction $=0.15$ ). Similarly, aortic valve mean gradients decreased significantly in both groups (men: from $41 \pm 15 \mathrm{mmHg}$ to $9 \pm 4 \mathrm{mmHg}$ immediately after TAVI and to $9 \pm 5 \mathrm{mmHg}$ at 6 and 12 months after TAVI, $p<0.001$; women: from $42 \pm 17 \mathrm{mmHg}$ to $9 \pm 5 \mathrm{mmHg}$ immediately after TAVI and to $10 \pm 5 \mathrm{mmHg}$ at 6 and 12 months, $p<0.001 ; p$ for interaction=0.74). The echocardiographic data of the overall population and according to sex at 6 months and 12 months follow-up are summarized in Supplemental Table 1.

The echocardiographic endpoints for the overall population and according to sex at 6 and 12 months after TAVI are summarized in Table 3. No significant differences were observed between men and women in terms of absolute reduction of indexed LV volumes and LV mass as well as improvement in LV function, nor if expressed as percentual change as compared to baseline. Moreover, a $\geq 10 \%$ reduction in indexed LV volumes and LV mass during follow-up was equally observed among men and women. The rates of increased aortic valve prosthetic mean gradient $(\geq 20 \mathrm{mmHg}$ ) were comparable between men and women at 6 months after TAVI, but were more common in women at 12 months follow-up ( $6 \%$ vs. $2 \%$, respectively, $p=0.041$ ). 
Table 3

Echocardiographic endpoints at 6 months and 12 months after transcatheter aortic valve implantation.

\begin{tabular}{|c|c|c|c|c|c|}
\hline Variable & & $\begin{array}{l}\text { Overall } \\
\text { population }\end{array}$ & Men & Women & $\begin{array}{l}\mathrm{p}- \\
\text { value }\end{array}$ \\
\hline \multirow[t]{2}{*}{ Absolute LVEDVi reduction, $\mathrm{ml} / \mathrm{m}^{2}$} & $\begin{array}{l}6 m \\
(n=439)\end{array}$ & $2.6 \pm 12.1$ & $\begin{array}{l}3.3 \pm \\
13.7\end{array}$ & $\begin{array}{l}1.9 \pm \\
10.1\end{array}$ & 0.21 \\
\hline & $\begin{array}{l}12 m \\
(n=408)\end{array}$ & $4.9 \pm 13.0$ & $\begin{array}{l}5.0 \pm \\
14.3\end{array}$ & $\begin{array}{l}4.7 \pm \\
11.4\end{array}$ & 0.86 \\
\hline \multirow[t]{2}{*}{ Absolute LVESVi reduction, $\mathrm{ml} / \mathrm{m}^{2}$} & $\begin{array}{l}6 m \\
(n=439)\end{array}$ & $2.7 \pm 8.0$ & $\begin{array}{l}3.2 \pm \\
8.6\end{array}$ & $2.1 \pm$ & 0.14 \\
\hline & $\begin{array}{l}12 m \\
(n=408)\end{array}$ & $4.0 \pm 9.1$ & $\begin{array}{l}4.0 \pm \\
9.9\end{array}$ & $\begin{array}{l}4.0 \pm \\
8.1\end{array}$ & 0.99 \\
\hline \multirow[t]{2}{*}{ Absolute LVEF improvement, \% } & $\begin{array}{l}6 m \\
(n=439)\end{array}$ & $2.6 \pm 8.8$ & $\begin{array}{l}2.6 \pm \\
8.8\end{array}$ & $\begin{array}{l}2.7 \pm \\
8.9\end{array}$ & 0.91 \\
\hline & $\begin{array}{l}12 m \\
(n=408)\end{array}$ & $3.5 \pm 9.0$ & $\begin{array}{l}3.0 \pm \\
9.1\end{array}$ & $\begin{array}{l}4.2 \pm \\
8.8\end{array}$ & 0.18 \\
\hline \multirow[t]{2}{*}{ Absolute LV mass index reduction, $\mathrm{g} / \mathrm{m}^{2}$} & $\begin{array}{l}6 m \\
(n=435)\end{array}$ & $13 \pm 22$ & $15 \pm 22$ & $12 \pm 21$ & 0.16 \\
\hline & $\begin{array}{l}12 m \\
(n=406)\end{array}$ & $20 \pm 24$ & $20 \pm 24$ & $20 \pm 25$ & 0.80 \\
\hline \multirow[t]{2}{*}{$\begin{array}{l}\text { LVEDVi reduction as percentage of } \\
\text { baseline, } \%\end{array}$} & $\begin{array}{l}6 m \\
(n=439)\end{array}$ & $2.5 \pm 24.0$ & $\begin{array}{l}3.2 \pm \\
23.9\end{array}$ & $\begin{array}{l}1.7 \pm \\
24.1\end{array}$ & 0.52 \\
\hline & $\begin{array}{l}12 m \\
(n=408)\end{array}$ & $6.8 \pm 25.3$ & $\begin{array}{l}6.5 \pm \\
24.3\end{array}$ & $\begin{array}{l}7.2 \pm \\
26.5\end{array}$ & 0.80 \\
\hline \multirow[t]{2}{*}{$\begin{array}{l}\text { LVESVi reduction as percentage of } \\
\text { baseline, \% }\end{array}$} & $\begin{array}{l}6 m \\
(n=439)\end{array}$ & $6.3 \pm 28.0$ & $\begin{array}{l}7.4 \pm \\
25.8\end{array}$ & $\begin{array}{l}5.2 \pm \\
30.2\end{array}$ & 0.41 \\
\hline & $\begin{array}{l}12 m \\
(n=408)\end{array}$ & $11.4 \pm 31.0$ & $\begin{array}{l}11.0 \pm \\
27.9\end{array}$ & $\begin{array}{l}11.8 \pm \\
34.3\end{array}$ & 0.78 \\
\hline \multirow[t]{2}{*}{$\begin{array}{l}\text { LV mass index reduction as percentage } \\
\text { of baseline, \% }\end{array}$} & $\begin{array}{l}6 m \\
(n=435)\end{array}$ & $9.8 \pm 16.6$ & $\begin{array}{l}10.7 \pm \\
15.9\end{array}$ & $\begin{array}{l}8.9 \pm \\
17.3\end{array}$ & 0.26 \\
\hline & $\begin{array}{l}12 m \\
(n=406)\end{array}$ & $15.0 \pm 16.5$ & $\begin{array}{l}14.8 \pm \\
15.6\end{array}$ & $\begin{array}{l}15.2 \pm \\
17.5\end{array}$ & 0.83 \\
\hline \multirow[t]{2}{*}{ LVEDVi reduction, $\mathrm{n}(\%)$} & $\begin{array}{l}6 m \\
(n=439)\end{array}$ & $261(60)$ & $\begin{array}{l}137 \\
(59)\end{array}$ & $\begin{array}{l}124 \\
(60)\end{array}$ & 0.95 \\
\hline & $\begin{array}{l}12 m \\
(n=408)\end{array}$ & $269(66)$ & $\begin{array}{l}138 \\
(64)\end{array}$ & $\begin{array}{l}131 \\
(68)\end{array}$ & 0.36 \\
\hline
\end{tabular}

Data are presented as mean $\pm S D$ and $n(\%) . A V=$ aortic valve, $L V=$ left ventricular, $L V E D V i=$ left ventricular end-diastolic volume index, LVEF = left ventricular ejection fraction, LVESVi = left ventricular end-systolic volume index. 


\begin{tabular}{|c|c|c|c|c|c|}
\hline Variable & & $\begin{array}{l}\text { Overall } \\
\text { population }\end{array}$ & Men & Women & $\begin{array}{l}\mathrm{p}- \\
\text { value }\end{array}$ \\
\hline \multirow[t]{2}{*}{ LVESVi reduction, n (\%) } & $\begin{array}{l}6 m \\
(n=439)\end{array}$ & $288(66)$ & $\begin{array}{l}154 \\
(67)\end{array}$ & $\begin{array}{l}134 \\
(64)\end{array}$ & 0.62 \\
\hline & $\begin{array}{l}12 m \\
(n=408)\end{array}$ & $283(69)$ & $\begin{array}{l}145 \\
(67)\end{array}$ & $\begin{array}{l}138 \\
(72)\end{array}$ & 0.30 \\
\hline \multirow[t]{2}{*}{ LVEF improvement, n (\%) } & $\begin{array}{l}6 m \\
(n=439)\end{array}$ & $274(62)$ & $\begin{array}{l}138 \\
(60)\end{array}$ & $\begin{array}{l}136 \\
(65)\end{array}$ & 0.22 \\
\hline & $\begin{array}{l}12 m \\
(n=408)\end{array}$ & $280(69)$ & $\begin{array}{l}143 \\
(66)\end{array}$ & $\begin{array}{l}137 \\
(71)\end{array}$ & 0.26 \\
\hline \multirow[t]{2}{*}{ LV mass index reduction, $\mathrm{n}(\%)$} & $\begin{array}{l}6 m \\
(n=435)\end{array}$ & $324(75)$ & $\begin{array}{l}175 \\
(77)\end{array}$ & $\begin{array}{l}149 \\
(72)\end{array}$ & 0.25 \\
\hline & $\begin{array}{l}12 m \\
(n=406)\end{array}$ & $337(83)$ & $\begin{array}{l}179 \\
(84)\end{array}$ & $\begin{array}{l}158 \\
(82)\end{array}$ & 0.56 \\
\hline \multirow[t]{2}{*}{$\geq 10 \%$ LVEDVi reduction, $\mathrm{n}(\%)$} & $\begin{array}{l}6 m \\
(n=439)\end{array}$ & $178(41)$ & $94(41)$ & $84(40)$ & 0.95 \\
\hline & $\begin{array}{l}12 m \\
(n=408)\end{array}$ & $200(49)$ & $\begin{array}{l}105 \\
(49)\end{array}$ & $95(50)$ & 0.86 \\
\hline \multirow[t]{2}{*}{$\geq 10 \%$ LVESVi reduction, $\mathrm{n}(\%)$} & $\begin{array}{l}6 \mathrm{~m} \\
(\mathrm{n}=439)\end{array}$ & $201(46)$ & $\begin{array}{l}109 \\
(47)\end{array}$ & $92(44)$ & 0.54 \\
\hline & $\begin{array}{l}12 m \\
(n=408)\end{array}$ & $234(57)$ & $\begin{array}{l}119 \\
(55)\end{array}$ & $\begin{array}{l}115 \\
(60)\end{array}$ & 0.33 \\
\hline \multirow[t]{2}{*}{$\geq 10 \%$ LV mass index reduction, $n(\%)$} & $\begin{array}{l}6 m \\
(n=435)\end{array}$ & $231(53)$ & $\begin{array}{l}122 \\
(54)\end{array}$ & $\begin{array}{l}109 \\
(53)\end{array}$ & 0.86 \\
\hline & $\begin{array}{l}12 m \\
(n=406)\end{array}$ & $271(67)$ & $\begin{array}{l}144 \\
(68)\end{array}$ & $\begin{array}{l}127 \\
(66)\end{array}$ & 0.70 \\
\hline \multirow[t]{2}{*}{$\geq 20 \%$ LV mass index regression, $n(\%)$} & $\begin{array}{l}6 m \\
(n=435)\end{array}$ & $117(27)$ & $61(27)$ & $56(27)$ & 0.94 \\
\hline & $\begin{array}{l}12 m \\
(n=406)\end{array}$ & $150(37)$ & $71(33)$ & $79(41)$ & 0.11 \\
\hline \multirow[t]{2}{*}{ AV mean gradient $\geq 20 \mathrm{mmHg}, \mathrm{n}(\%)$} & $\begin{array}{l}6 m \\
(n=434)\end{array}$ & $15(4)$ & $6(3)$ & $9(4)$ & 0.33 \\
\hline & $\begin{array}{l}12 m \\
(n=406)\end{array}$ & $15(4)$ & $4(2)$ & $11(6)$ & 0.041 \\
\hline$\geq 5 \%$ LVEF reduction, $\mathrm{n}(\%)$ & $\begin{array}{l}6 m \\
(n=439)\end{array}$ & $70(16)$ & $35(15)$ & 35 (17) & 0.63 \\
\hline
\end{tabular}

Data are presented as mean $\pm S D$ and $n(\%) . A V=$ aortic valve, $L V=$ left ventricular, $L V E D V i=$ left ventricular end-diastolic volume index, LVEF = left ventricular ejection fraction, LVESVi = left ventricular end-systolic volume index. 


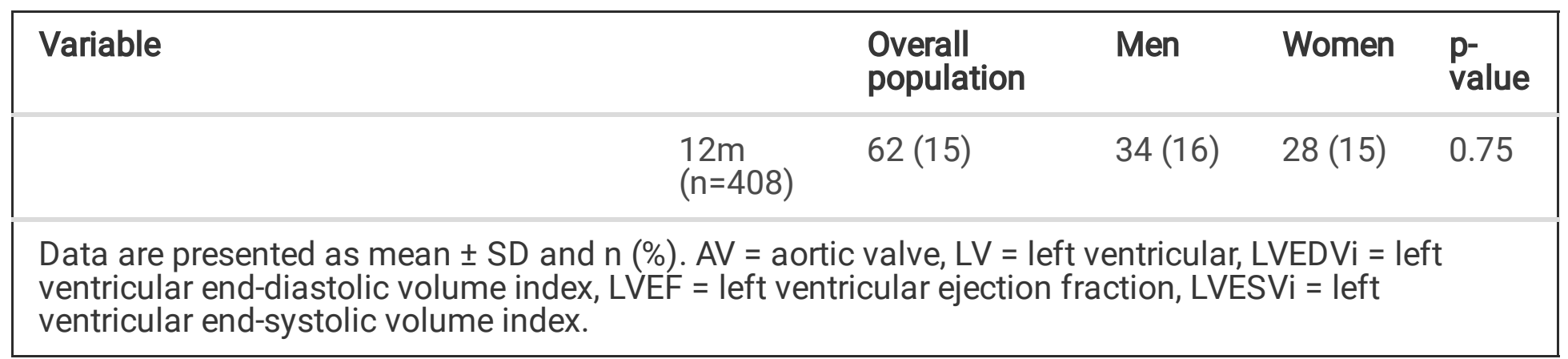

\section{Clinical outcomes and impact of sex on LV reverse remodeling}

During a median follow-up of 2.8 [IQR 1.9-4.3] years after the 6 or 12 months echocardiographic examination following TAVI, 181 (39\%) patients died of which 103 were men and 78 women. Overall, the cumulative survival rates were $90 \%$ at 1 year and $46 \%$ at 5 years. Kaplan-Meier analysis demonstrated superior long-term survival in women versus men (log-rank $\chi^{2}: 5.10, p=0.024$, Figure 4). Moreover, multivariable Cox proportional hazard regression analysis demonstrated that male sex was independently associated with an increased risk of all-cause mortality after adjusting for age, cardiac risk factors, comorbidities, and pre-TAVI LVEF (HR 1.423, 95\% Cl: 1.039-1.951, p=0.028, Table 4). In addition, there was no significant association between the percentual LV mass index reduction and sex (as an interaction term) versus outcomes $(p=0.64)$.

Table 4

Uni- and multivariable Cox regression analysis for all-cause mortality.

\begin{tabular}{|c|c|c|c|c|c|c|}
\hline \multirow[b]{2}{*}{ Parameter } & \multicolumn{3}{|c|}{ Univariable } & \multicolumn{3}{|c|}{ Multivariable } \\
\hline & HR & $95 \% \mathrm{Cl}$ & p-value & HR & $95 \% \mathrm{Cl}$ & p-value \\
\hline Male sex & 1.402 & $1.044-1.883$ & 0.025 & 1.423 & $1.039-1.951$ & 0.028 \\
\hline $1 \%$ LV mass index reduction & 0.992 & $0.984-1.000$ & 0.065 & 0.992 & 0.983-1.000 & 0.060 \\
\hline
\end{tabular}

\section{Discussion}

In patients with severe AS, men and women showed a similar reduction in LV volumes and LV mass as well as improvement in LVEF after TAVI, despite smaller LV volumes and mass in women before TAVI. In addition, women showed better survival after TAVI as compared to men. However, the survival benefit of women is not explained by sex differences in LV reverse remodeling after TAVI.

\section{Sex differences in LV reverse remodeling after TAVI}


AS causes an increase in LV afterload to which the myocardium responds with LV hypertrophy in order to normalize wall stress and maintain cardiac output $[15,28]$. Aortic valve replacement directly relieves the increased LV afterload, leading to lower aortic valve gradients and LV reverse remodeling with LV mass regression, reduction in LV volumes, and improvement in LV systolic function [29, 30]. Similarly, the current study showed that patients with severe AS had a significant reduction in LV volumes and LV mass with an improvement of LVEF during the first year after TAVI.

Sex-related differences in LV remodeling in response to the increased pressure overload caused by AS have been noted. Before aortic valve replacement, women show smaller LV volumes and LV mass as compared to men for a similar degree of AS severity [28,31]. However, at follow-up after TAVI there is a similar magnitude of reduction in LV mass index and volumes and improvement in LVEF in men and women, as shown in the present study. Similarly, a study including 100 patients with severe AS treated with surgical aortic valve replacement or TAVI did not show sex differences in LV reverse remodeling at 6 months using cardiac magnetic resonance imaging [16]. In contrast, a study evaluating 100 patients with severe AS treated with TAVI reported a significant improvement in LVEF among women at 3 months after TAVI, but not in men [17]. In addition, Ninomiya et al. [32] showed in a study of 208 patients with severe AS treated with TAVI, that the incidence of LV reverse remodeling (defined as a reduction of LV endsystolic volume $>15 \%$ evaluated by echocardiography at 3 months after TAVI) was significantly higher in men versus women. These results show the heterogeneity in defining LV reverse remodeling after TAVI which challenges the comparison across studies.

\section{Sex differences in outcomes after TAVI and association with LV reverse remodeling}

This study demonstrated superior survival in women after TAVI in line with previous reports [10, 11]. Several factors have been suggested for the sex-related differences in outcomes after TAVI, including sex differences in (paravalvular) aortic regurgitation of the transcatheter valve due to patient-prothesis mismatch, more co-existent comorbidities in men, the timing of referral/treatment of patients with severe AS (women earlier referral than men), and the longer life expectancy in women versus men [12].

Another explanation may be the sex-specific myocardial remodeling in AS. Previously, our group examined the impact of sex on LV remodeling before TAVI and reported that the outcome benefits of women after TAVI were not associated with sex differences in LV remodeling [33]. However, the impact of the potential sex-related differences in LV reverse remodeling after TAVI on outcomes remained unexplored. In the current study, no significant interaction was observed between sex and LV reverse remodeling and its association with outcomes. It is important to note that men remained with larger LV volumes and LV mass index as compared to women at follow-up after TAVI, which could indicate that men have a more pronounced LV hypertrophic response to severe AS that is not fully recovered when the pressure overload is relieved. This might be related to the observed increased risk for all-cause mortality [34]. However, the interaction term of sex and the magnitude in reduction of LV mass index and LV volumes was not independently associated with all-cause mortality. 


\section{Future studies}

Further research is necessary to confirm our observations and to elucidate the potential causes of the sex differences in outcomes observed after aortic valve replacement. Currently, the prospective, multicenter, Randomized researcH in womEn all comers wlth Aortic stenosis (RHEIA) trial is being performed to evaluate the safety and efficacy of surgical aortic valve replacement versus TAVI specifically in women with severe AS [35]. The results of the RHEIA trial may provide important data for the optimal therapeutic management of severe AS in women.

\section{Limitations}

Several limitations should be acknowledged. This is a single-center retrospective analysis with inherent limitations related to the study design. Second, only patients with echocardiographic follow-up after TAVI were included in the current analysis which may have introduced a selection bias. In addition, the echocardiographic follow-up was limited to 1 year, whereas LV reverse remodeling is a process that may continue beyond 1 year after aortic valve replacement. Last, arterial afterload (i.e. co-existent arterial hypertension) and myocardial fibrosis may affect LV reverse remodeling after TAVI. However, this study used echocardiography to assess LV reverse remodeling and could detect the presence and extent of myocardial fibrosis.

\section{Conclusion}

Patients with severe AS had a significant improvement in LVEF, reduction in LV volumes and LV mass index at 6 and 12 months after TAVI, without significant differences between men and women over time. Women showed better survival after TAVI as compared to men. However, the interaction between the percentual LV mass index reduction and sex was not associated with survival. These data suggest that the superior outcomes noted in women after TAVI are not associated with sex differences in LV reverse remodeling.

\section{Declarations}

\section{Funding}

Jan Stassen received funding from the European Society of Cardiology in form of an ESC Training Grant (App000064741). Stephan M. Pio received funding from the European Society of Cardiology in form of an ESC Training Grant (T-2018-17405).

\section{Conflict of interest}

The department of Cardiology, Leiden University Medical Center, Leiden, the Netherlands has received unrestricted research grants from Abbot Vascular, Bayer, Bioventrix, GE Healthcare, lonis, Medtronic, Biotronik, Boston Scientific, and Edwards Lifesciences. Jeroen J. Bax and Nina Ajmone Marsan received speaker fees from Abbot Vascular. Victoria Delgado received speaker fees from Abbott Vascular, Edwards 
Lifesciences, GE Healthcare, MSD, Novartis and Medtronic. Juhani Knuuti received consultancy fees from GE Healthcare and AstraZeneca and speaker fees from GE Healthcare, Bayer, Lundbeck, BoehringerIngelheim, Pfizer and Merck, outside of the submitted work. All other authors have reported that they have no relationships relevant to the contents of this paper to disclose.

\section{Availability of data}

The data underlying this article will be shared on reasonable request to the corresponding author.

\section{Authors' contributions}

All authors contributed and participated in the preparation of the manuscript as follows: Kuneman: Conceptualization, Methodology, Data curation, Formal analysis, Visualization, Writing - original draft preparation, editing. Butcher: Conceptualization, Formal analysis, Writing- reviewing and editing; Stassen: Conceptualization, Methodology, Writing - review \& editing; Singh, van der Kley: Data curation, Writingreviewing and editing; Pio: Conceptualization, Writing - review \& editing; Ajmone, Knuuti, Bax, Delgado: Conceptualization, Methodology, Writing- reviewing and editing, Supervision. All authors carefully reviewed and approved the final version of the article.

\section{Ethics approval}

This retrospective analysis complies with the Declaration of Helsinki and was approved by the institutional review board of the Leiden University Medical Center Leiden, The Netherlands.

\section{Consent to participate}

The institutional review board of the Leiden University Medical Center Leiden, The Netherlands waived the need for written informed consent due to the retrospective study design.

\section{References}

1. Rosenhek R, Zilberszac R, Schemper M, Czerny M, Mundigler G, Graf S, Bergler-Klein J, Grimm M, Gabriel H, Maurer G (2010) Natural history of very severe aortic stenosis. Circulation 121:151-156. https://doi.org/10.1161/CIRCULATIONAHA.109.894170

2. Tang L, Gossl M, Ahmed A, Garberich R, Bradley SM, Niikura H, Witt D, Pedersen WR, Bae R, Lesser JR, Harris KM, Sun B, Mudy K, Sorajja P (2018) Contemporary Reasons and Clinical Outcomes for Patients With Severe, Symptomatic Aortic Stenosis Not Undergoing Aortic Valve Replacement. Circ Cardiovasc Interv 11:e007220. https://doi.org/10.1161/CIRCINTERVENTIONS.118.007220

3. Kang DH, Park SJ, Lee SA, Lee S, Kim DH, Kim HK, Yun SC, Hong GR, Song JM, Chung CH, Song JK, Lee JW, Park SW (2020) Early Surgery or Conservative Care for Asymptomatic Aortic Stenosis. N Engl J Med 382:111-119. https://doi.org/10.1056/NEJMoa1912846 
4. Otto CM, Nishimura RA, Bonow RO, Carabello BA, Erwin JP 3, Gentile F, Jneid H, Krieger EV, Mack M, McLeod C, O'Gara PT, Rigolin VH, Sundt TM 3, Thompson A, Toly C (2021) 2020 ACC/AHA Guideline for the Management of Patients With Valvular Heart Disease: A Report of the American College of Cardiology/American Heart Association Joint Committee on Clinical Practice Guidelines. Circulation 143:e72-e227. https://doi.org/10.1161/CIR.0000000000000923

5. Leon MB, Smith CR, Mack M, Miller DC, Moses JW, Svensson LG, Tuzcu EM, Webb JG, Fontana GP, Makkar RR, Brown DL, Block PC, Guyton RA, Pichard AD, Bavaria JE, Herrmann HC, Douglas PS, Petersen JL, Akin JJ, Anderson WN, Wang D, Pocock S, Investigators PT (2010) Transcatheter aorticvalve implantation for aortic stenosis in patients who cannot undergo surgery. $\mathrm{N}$ Engl $\mathrm{J}$ Med 363:1597-1607. https://doi.org/10.1056/NEJMoa1008232

6. Siontis GCM, Overtchouk P, Cahill TJ, Modine T, Prendergast B, Praz F, Pilgrim T, Petrinic T, Nikolakopoulou A, Salanti G, Sondergaard L, Verma S, Juni P, Windecker S (2019) Transcatheter aortic valve implantation vs. surgical aortic valve replacement for treatment of symptomatic severe aortic stenosis: an updated meta-analysis. Eur Heart J 40:3143-3153.

https://doi.org/10.1093/eurheartj/ehz275

7. Mack MJ, Leon MB, Thourani VH, Makkar R, Kodali SK, Russo M, Kapadia SR, Malaisrie SC, Cohen DJ, Pibarot P, Leipsic J, Hahn RT, Blanke P, Williams MR, McCabe JM, Brown DL, Babaliaros V, Goldman S, Szeto WY, Genereux P, Pershad A, Pocock SJ, Alu MC, Webb JG, Smith CR, Investigators P (2019) Transcatheter Aortic-Valve Replacement with a Balloon-Expandable Valve in Low-Risk Patients. N Engl J Med 380:1695-1705. https://doi.org/10.1056/NEJMoa1814052

8. Leon MB, Smith CR, Mack MJ, Makkar RR, Svensson LG, Kodali SK, Thourani VH, Tuzcu EM, Miller DC, Herrmann HC, Doshi D, Cohen DJ, Pichard AD, Kapadia S, Dewey T, Babaliaros V, Szeto WY, Williams MR, Kereiakes D, Zajarias A, Greason KL, Whisenant BK, Hodson RW, Moses JW, Trento A, Brown DL, Fearon WF, Pibarot P, Hahn RT, Jaber WA, Anderson WN, Alu MC, Webb JG, Investigators P (2016) Transcatheter or Surgical Aortic-Valve Replacement in Intermediate-Risk Patients. N Engl J Med 374:1609-1620. https://doi.org/10.1056/NEJMoa1514616

9. Williams M, Kodali SK, Hahn RT, Humphries KH, Nkomo VT, Cohen DJ, Douglas PS, Mack M, McAndrew TC, Svensson L, Thourani VH, Tuzcu EM, Weissman NJ, Kirtane AJ, Leon MB (2014) Sexrelated differences in outcomes after transcatheter or surgical aortic valve replacement in patients with severe aortic stenosis: Insights from the PARTNER Trial (Placement of Aortic Transcatheter Valve). J Am Coll Cardiol 63:1522-1528. https://doi.org/10.1016/j.jacc.2014.01.036

10. Biere L, Launay M, Pinaud F, Hamel JF, Eltchaninoff H, lung B, Laskar M, Leguerrier A, Gilard M, Furber A (2015) Influence of sex on mortality and perioperative outcomes in patients undergoing TAVR: insights from the FRANCE 2 registry. J Am Coll Cardiol 65:755-757.

https://doi.org/10.1016/j.jacc.2014.11.044

11. Chandrasekhar J, Dangas G, Yu J, Vemulapalli S, Suchindran S, Vora AN, Baber U, Mehran R, Registry SAT (2016) Sex-Based Differences in Outcomes With Transcatheter Aortic Valve Therapy: TVT Registry From 2011 to 2014. J Am Coll Cardiol 68:2733-2744.

https://doi.org/10.1016/j.jacc.2016.10.041

Page $17 / 24$ 
12. Saad M, Nairooz R, Pothineni NVK, Almomani A, Kovelamudi S, Sardar P, Katz M, Abdel-Wahab M, Bangalore S, Kleiman NS, Block PC, Abbott JD (2018) Long-Term Outcomes With Transcatheter Aortic Valve Replacement in Women Compared With Men: Evidence From a Meta-Analysis. JACC Cardiovasc Interv 11:24-35. https://doi.org/10.1016/j.jcin.2017.08.015

13. O'Connor SA, Morice MC, Gilard M, Leon MB, Webb JG, Dvir D, Rodes-Cabau J, Tamburino C, Capodanno D, D'Ascenzo F, Garot P, Chevalier B, Mikhail GW, Ludman PF (2015) Revisiting Sex Equality With Transcatheter Aortic Valve Replacement Outcomes: A Collaborative, Patient-Level Meta-Analysis of 11,310 Patients. J Am Coll Cardiol 66:221-228.

https://doi.org/10.1016/j.jacc.2015.05.024

14. Otto CM, Prendergast B (2014) Aortic-valve stenosis-from patients at risk to severe valve obstruction. N Engl J Med 371:744-756. https://doi.org/10.1056/NEJMra1313875

15. Grossman W, Jones D, McLaurin LP (1975) Wall stress and patterns of hypertrophy in the human left ventricle. J Clin Invest 56:56-64. https://doi.org/10.1172/JCI108079

16. Dobson LE, Fairbairn TA, Musa TA, Uddin A, Mundie CA, Swoboda PP, Ripley DP, McDiarmid AK, Erhayiem B, Garg P, Malkin CJ, Blackman DJ, Sharples LD, Plein S, Greenwood JP (2016) Sex-related differences in left ventricular remodeling in severe aortic stenosis and reverse remodeling after aortic valve replacement: A cardiovascular magnetic resonance study. Am Heart J 175:101-111. https://doi.org/10.1016/j.ahj.2016.02.010

17. Stangl V, Baldenhofer G, Knebel F, Zhang K, Sanad W, Spethmann S, Grubitzsch H, Sander M, Wernecke KD, Baumann G, Stangl K, Laule M (2012) Impact of gender on three-month outcome and left ventricular remodeling after transfemoral transcatheter aortic valve implantation. Am $\mathrm{J}$ Cardiol 110:884-890. https://doi.org/10.1016/j.amjcard.2012.04.063

18. Petrov G, Regitz-Zagrosek V, Lehmkuhl E, Krabatsch T, Dunkel A, Dandel M, Dworatzek E, Mahmoodzadeh S, Schubert C, Becher E, Hampl H, Hetzer R (2010) Regression of myocardial hypertrophy after aortic valve replacement: faster in women? Circulation 122:S23-28. https://doi.org/10.1161/CIRCULATIONAHA.109.927764

19. Chen SC, Leu HB, Chang HH, Chen IM, Chen PL, Lin SM, Chen YH (2020) Women had favourable reverse left ventricle remodelling after TAVR. Eur J Clin Invest 50:e13183. https://doi.org/10.1111/eci.13183

20. Delgado V, Ng AC, van de Veire NR, van der Kley F, Schuijf JD, Tops LF, de Weger A, Tavilla G, de Roos A, Kroft LJ, Schalij MJ, Bax JJ (2010) Transcatheter aortic valve implantation: role of multi-detector row computed tomography to evaluate prosthesis positioning and deployment in relation to valve function. Eur Heart J 31:1114-1123. https://doi.org/10.1093/eurheartj/ehq018

21. Otto CM, Kumbhani DJ, Alexander KP, Calhoon JH, Desai MY, Kaul S, Lee JC, Ruiz CE, Vassileva CM (2017) 2017 ACC Expert Consensus Decision Pathway for Transcatheter Aortic Valve Replacement in the Management of Adults With Aortic Stenosis: A Report of the American College of Cardiology Task Force on Clinical Expert Consensus Documents. J Am Coll Cardiol 69:1313-1346. https://doi.org/10.1016/j.jacc.2016.12.006 
22. Lancellotti P, Pibarot P, Chambers J, Edvardsen T, Delgado V, Dulgheru R, Pepi M, Cosyns B, Dweck MR, Garbi M, Magne J, Nieman K, Rosenhek R, Bernard A, Lowenstein J, Vieira ML, Rabischoffsky A, Vyhmeister RH, Zhou X, Zhang Y, Zamorano JL, Habib G (2016) Recommendations for the imaging assessment of prosthetic heart valves: a report from the European Association of Cardiovascular Imaging endorsed by the Chinese Society of Echocardiography, the Inter-American Society of Echocardiography, and the Brazilian Department of Cardiovascular Imaging. Eur Heart J Cardiovasc Imaging 17:589-590. https://doi.org/10.1093/ehjci/jew025

23. Lang RM, Badano LP, Mor-Avi V, Afilalo J, Armstrong A, Ernande L, Flachskampf FA, Foster E, Goldstein SA, Kuznetsova T, Lancellotti P, Muraru D, Picard MH, Rietzschel ER, Rudski L, Spencer KT, Tsang W, Voigt JU (2015) Recommendations for cardiac chamber quantification by echocardiography in adults: an update from the American Society of Echocardiography and the European Association of Cardiovascular Imaging. J Am Soc Echocardiogr 28:1-39e14. https://doi.org/10.1016/j.echo.2014.10.003

24. Ganau A, Devereux RB, Roman MJ, de Simone G, Pickering TG, Saba PS, Vargiu P, Simongini I, Laragh JH (1992) Patterns of left ventricular hypertrophy and geometric remodeling in essential hypertension. J Am Coll Cardiol 19:1550-1558. https://doi.org/10.1016/0735-1097(92)90617-v

25. Baumgartner $H$, Hung J, Bermejo J, Chambers JB, Edvardsen T, Goldstein S, Lancellotti P, LeFevre M, Miller F Jr, Otto CM (2017) Recommendations on the Echocardiographic Assessment of Aortic Valve Stenosis: A Focused Update from the European Association of Cardiovascular Imaging and the American Society of Echocardiography. J Am Soc Echocardiogr 30:372-392.

https://doi.org/10.1016/j.echo.2017.02.009

26. Zoghbi WA, Asch FM, Bruce C, Gillam LD, Grayburn PA, Hahn RT, Inglessis I, Islam AM, Lerakis S, Little SH, Siegel RJ, Skubas N, Slesnick TC, Stewart WJ, Thavendiranathan P, Weissman NJ, Yasukochi S, Zimmerman KG (2019) Guidelines for the Evaluation of Valvular Regurgitation After Percutaneous Valve Repair or Replacement: A Report from the American Society of Echocardiography Developed in Collaboration with the Society for Cardiovascular Angiography and Interventions, Japanese Society of Echocardiography, and Society for Cardiovascular Magnetic Resonance. J Am Soc Echocardiogr 32:431-475. https://doi.org/10.1016/j.echo.2019.01.003

27. Ngo A, Hassager C, Thyregod HGH, Sondergaard L, Olsen PS, Steinbruchel D, Hansen PB, Kjaergaard $J$, Winther-Jensen M, Ihlemann N (2018) Differences in left ventricular remodelling in patients with aortic stenosis treated with transcatheter aortic valve replacement with corevalve prostheses compared to surgery with porcine or bovine biological prostheses. Eur Heart J Cardiovasc Imaging 19:39-46. https://doi.org/10.1093/ehjci/jew321

28. Aurigemma GP, Silver KH, McLaughlin M, Mauser J, Gaasch WH (1994) Impact of chamber geometry and gender on left ventricular systolic function in patients $>60$ years of age with aortic stenosis. Am J Cardiol 74:794-798. https://doi.org/10.1016/0002-9149(94)90437-5

29. Chau KH, Douglas PS, Pibarot P, Hahn RT, Khalique OK, Jaber WA, Cremer P, Weissman NJ, Asch FM, Zhang Y, Gertz ZM, Elmariah S, Clavel MA, Thourani VH, Daubert M, Alu MC, Leon MB, Lindman BR (2020) Regression of Left Ventricular Mass After Transcatheter Aortic Valve Replacement: The 
PARTNER Trials and Registries. J Am Coll Cardiol 75:2446-2458.

https://doi.org/10.1016/j.jacc.2020.03.042

30. Magalhaes MA, Koifman E, Torguson R, Minha S, Gai J, Kiramijyan S, Escarcega RO, Baker NC, Wang Z, Goldstein S, Asch F, Satler LF, Pichard AD, Waksman R (2015) Outcome of Left-Sided Cardiac Remodeling in Severe Aortic Stenosis Patients Undergoing Transcatheter Aortic Valve Implantation. Am J Cardiol 116:595-603. https://doi.org/10.1016/j.amjcard.2015.05.018

31. Carroll JD, Carroll EP, Feldman T, Ward DM, Lang RM, McGaughey D, Karp RB (1992) Sex-associated differences in left ventricular function in aortic stenosis of the elderly. Circulation 86:1099-1107. https://doi.org/10.1161/01.cir.86.4.1099

32. Ninomiya R, Orii M, Fujiwara J, Yoshizawa M, Nakajima Y, Ishikawa Y, Kumagai A, Fusazaki T, Tashiro A, Kin H, Yoshioka K, Morino Y (2020) Sex-Related Differences in Cardiac Remodeling and Reverse Remodeling After Transcatheter Aortic Valve Implantation in Patients with Severe Aortic Stenosis in a Japanese Population. Int Heart J 61:961-969. https://doi.org/10.1536/ihj.20-154

33. Kuneman JH, Singh GK, Milhorini Pio S, Hirasawa K, Hautemann D, van der Kley F, Ajmone Marsan N, Knuuti J, Delgado V, Bax JJ (2021) Sex differences in left ventricular remodelling in patients with severe aortic valve stenosis. Eur Heart J Cardiovasc Imaging. https://doi.org/10.1093/ehjci/jeab174

34. Gonzales H, Douglas PS, Pibarot P, Hahn RT, Khalique OK, Jaber WA, Cremer P, Weissman NJ, Asch FM, Zhang Y, Gertz ZM, Elmariah S, Clavel MA, Thourani VH, Daubert M, Alu MC, Leon MB, Lindman BR (2020) Left Ventricular Hypertrophy and Clinical Outcomes Over 5 Years After TAVR: An Analysis of the PARTNER Trials and Registries. JACC Cardiovasc Interv 13:1329-1339. https://doi.org/10.1016/j.jcin.2020.03.011

35. Eltchaninoff H, Bonaros N, Prendergast B, Nietlispach F, Vasa-Nicotera M, Chieffo A, Pibarot P, Bramlage P, Sykorova L, Kurucova J, Bax JJ, Windecker S, Dumonteil N, Tchetche D (2020) Rationale and design of a prospective, randomized, controlled, multicenter study to evaluate the safety and efficacy of transcatheter heart valve replacement in female patients with severe symptomatic aortic stenosis requiring aortic valve intervention (Randomized researcH in womEn all comers wlth Aortic stenosis [RHEIA] trial). Am Heart J 228:27-35. https://doi.org/10.1016/j.ahj.2020.06.016

\section{Figures}


A

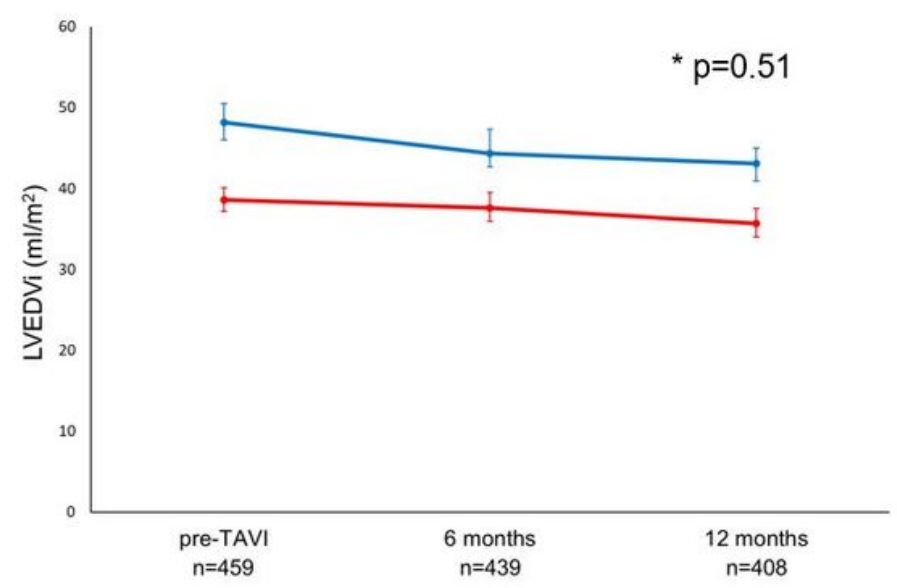

C

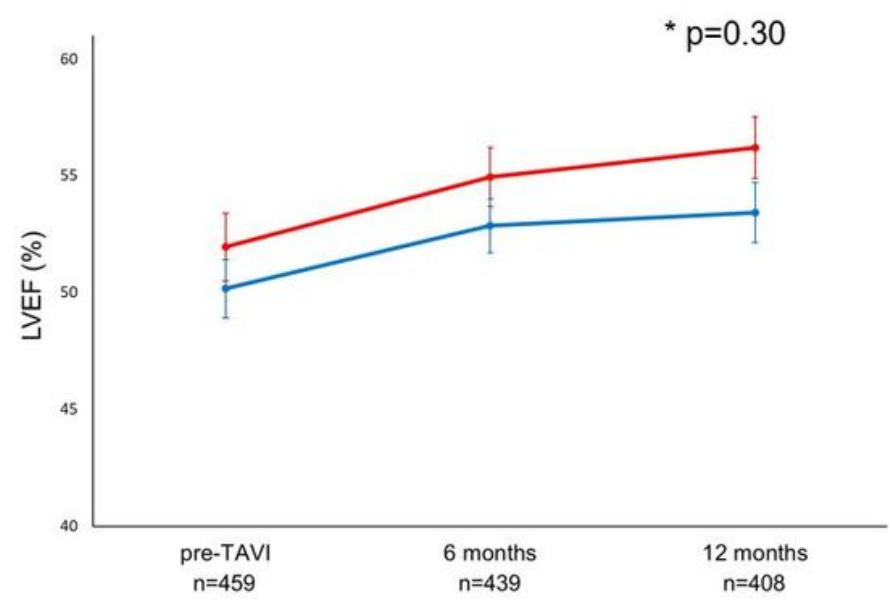

B

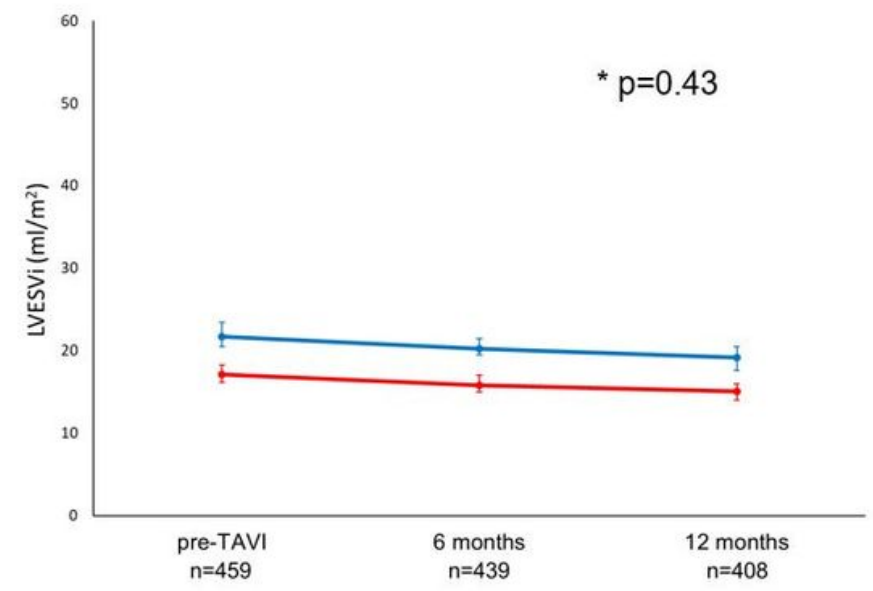

D

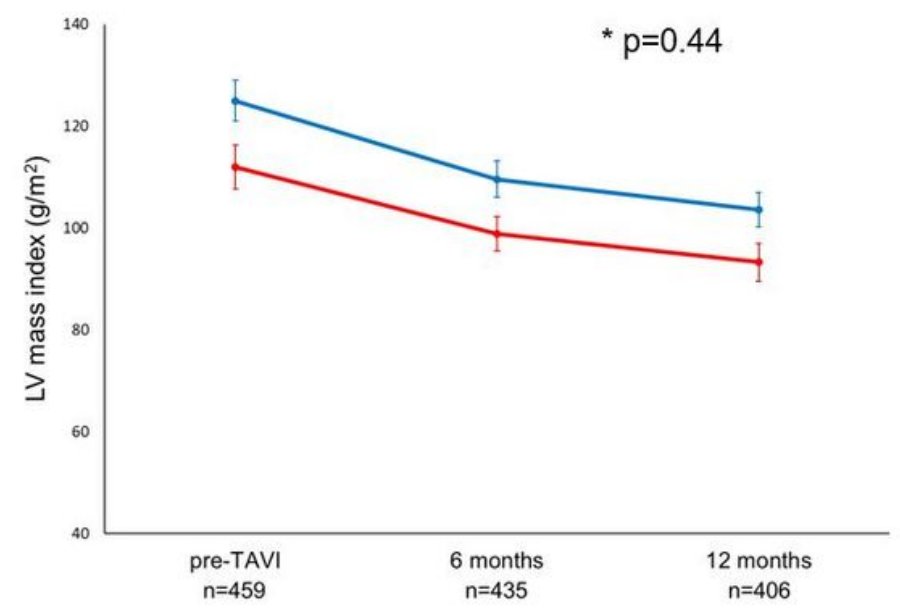

\section{Figure 1}

Left ventricular reverse remodeling in men (blue) and women (red) after transcatheter aortic valve implantation.

Changes in left ventricular end-diastolic volume index (LVEDVi, panel A), left ventricular end-systolic volume index (LVESVi, panel B), left ventricular ejection fraction (LVEF, panel C), and left ventricular mass index (panel D) from baseline to 6 months and 12 months follow-up after transcatheter aortic valve implantation. * shows p-value for interaction between men and women over time. Error bars indicate $95 \%$ confidence intervals. 
A

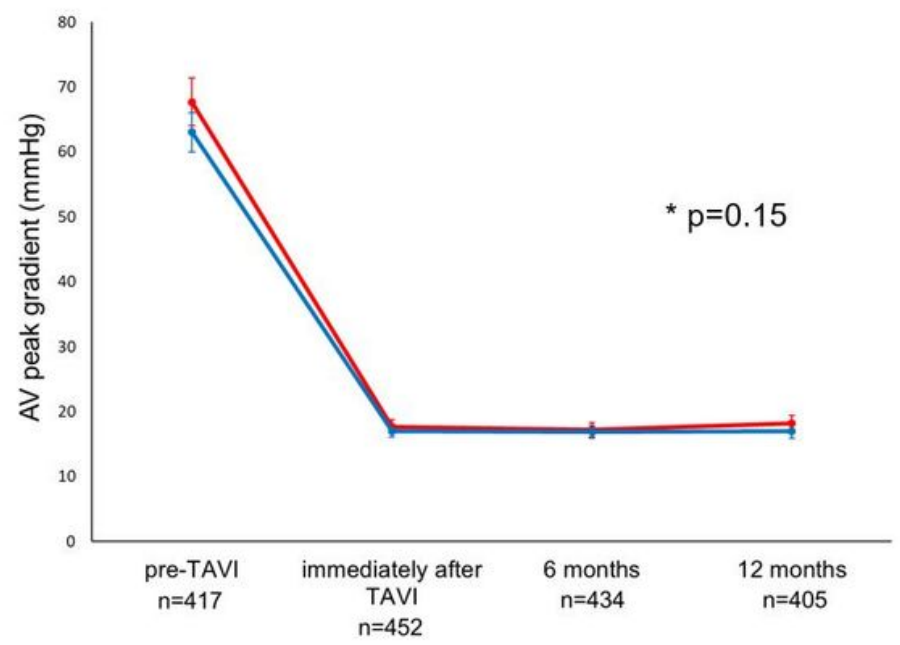

B

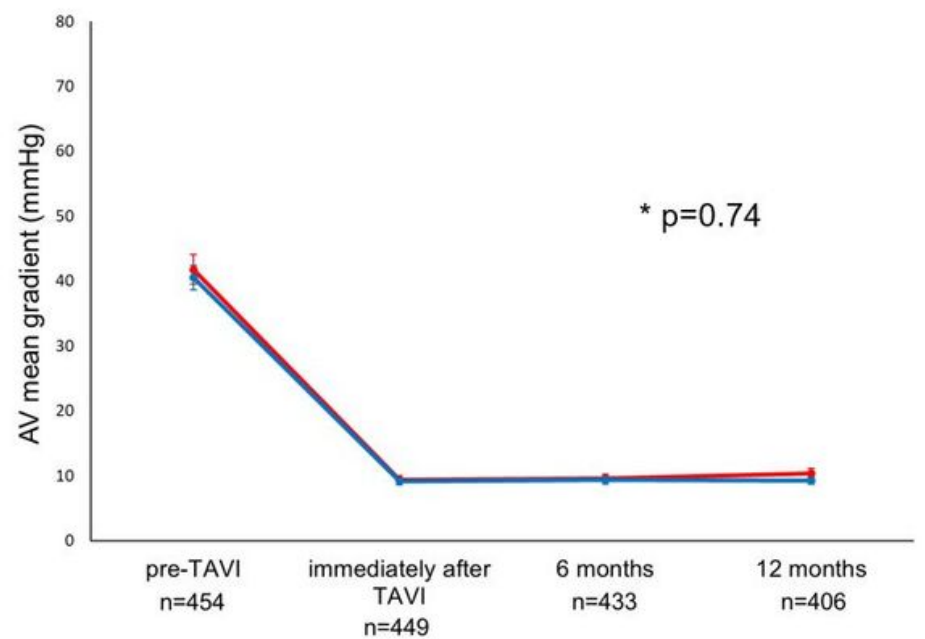

Figure 2

Aortic valve gradients of men (blue) and women (red) before transcatheter aortic valve implantation and during follow-up.

Changes in aortic valve peak gradient (panel A) and aortic valve mean gradient (panel B) from baseline to immediately after transcatheter aortic valve implantation and to 6 and 12 months follow-up. * shows pvalue for interaction between men and women over time. Error bars indicate $95 \%$ confidence intervals. 


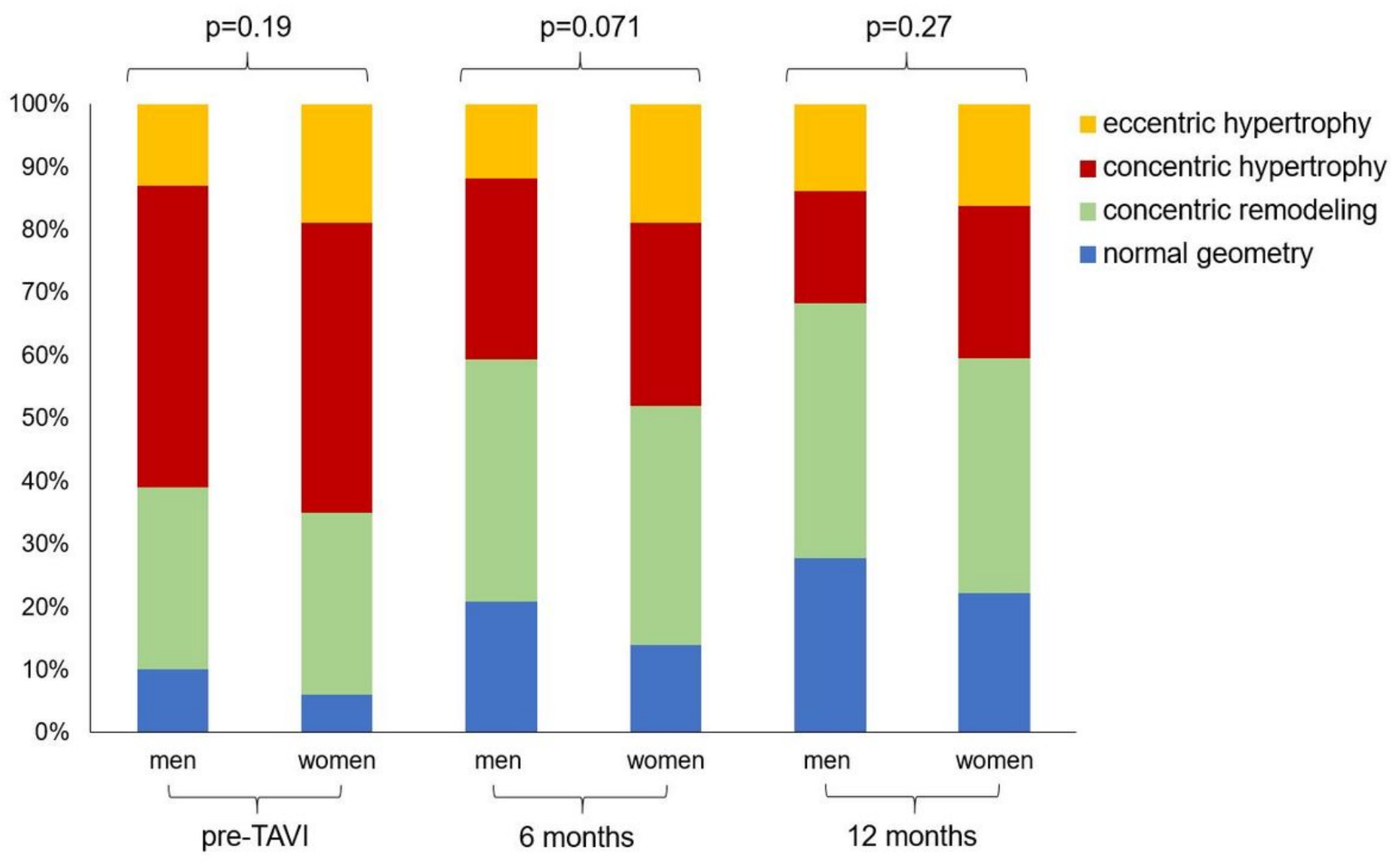

Figure 3

Distribution of the left ventricular remodeling patterns among men and women before transcatheter aortic valve implantation and at 6 and 12 months follow-up. 


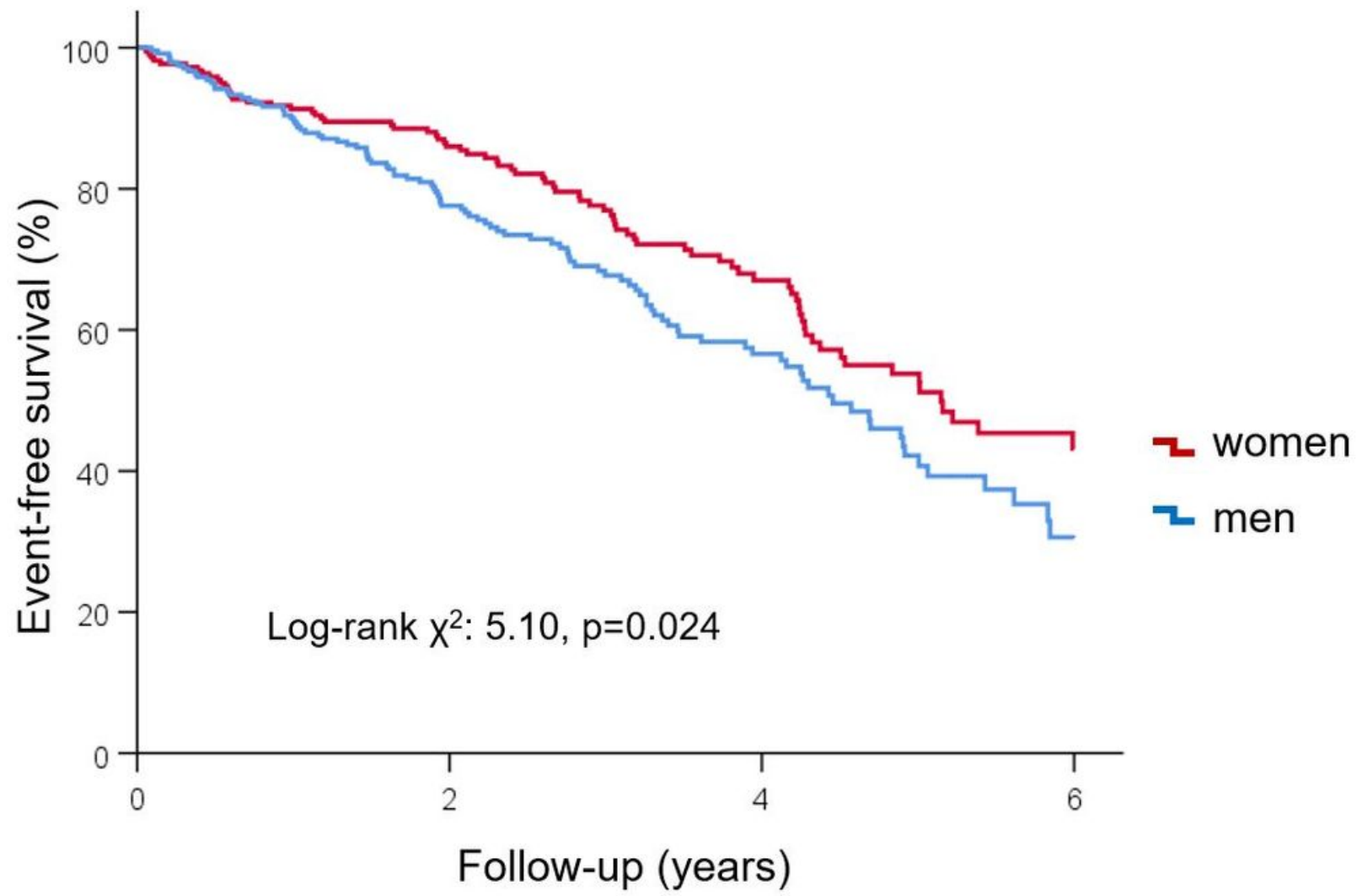

Number at risk

\begin{tabular}{l|l|l|l|l} 
women & 219 & 165 & 71 & 20 \\
\hline men & 240 & 159 & 64 & 12
\end{tabular}

Figure 4

Kaplan-Meier curves demonstrating the event-free survival for all-cause mortality according to sex.

\section{Supplementary Files}

This is a list of supplementary files associated with this preprint. Click to download.

- Graphicalabstract.pptx

- Supplementaldata.docx 